\title{
Free Vibration of Circular Plates with Elastic Edge Support and Resting on an Elastic Foundation
}

\author{
L. Bhaskara Rao \\ School of Mechanical and Building Sciences, VIT University, Chennai Campus, Vandalur-Kelambakkam Road, \\ Chennai-600127, India

\section{Kameswara Rao} \\ Department of Mechanical Engineering, TKR College of Engineering and Technology. Medbowli, Meerpet, Sa- \\ roornagar, Hyderabd-500 079, A.P., India
}

\section{(Received 2 April 2011; Revised 22 August 2012; Accepted 11 September 2012)}

An analytical study is presented for the vibration characteristics of thin circular plates with rotational elastic edge support and resting on an elastic foundation. The Winkler model was used to describe this foundation. The exact analytical method is used to derive the frequency equation of the plate with elastic support and resting on this foundation. Parametric investigations of the vibration of circular plates with an restrained elastically edge against rotation and resting on an elastic foundation have been carried out with respect to various rotational stiffness parameters, foundation stiffness parameters, and boundary conditions. Twelve vibration modes are presented. The location of the stepped region with respect to rotational restraint changed very little and moved from the range of $0.01426^{\dagger}$ [10.006 $2048^{\ddagger}$ ]-1 134.227 [10.025 94] to 0.01758 [14.001 133]-6 352.958 2 [14.658 356]. Here, ${ }^{\dagger}$ represents the rotational spring stiffness ratio, and ${ }^{\ddagger}$ represents the foundation stiffness ratio. Extensive data are tabulated so that pertinent conclusions can be arrived at on the influence of rotational edge restraint and the foundation parameters of a Winkler foundation on the natural frequencies of uniform thin isotropic circular plates.

\section{NOMENCLATURE}

$h$
$a$
$\nu$
$E$
$\rho$
$W(r, \theta)$
$D$
$K_{R}$
$K_{w}$
$R$
$\xi$
$\omega_{m n}$
$\lambda_{m n}$
$\lambda_{m n}^{*}$
$m, n$

ature shows that very few studies exist on circular plates resting on an elastic foundation. Wang and Wang, who observed the switching between axisymmetric and asymmetric vibration modes, recently investigated the effect of internal elastic translational supports. ${ }^{2}$

The vibration characteristics of plates resting on an elastic medium are different from those of the plates supported only on the boundary. Leissa discussed the vibration of a plate supported laterally by an elastic foundation. ${ }^{3}$ Leissa also deduced that the effect of a Winkler foundation merely increases the square of the natural frequency of the plate by a constant. Salari et al. arrived at the same conclusion. ${ }^{4}$ Ascione and Grimaldi studied unilateral frictionless contact between a circular plate and a Winkler foundation using a variational formulation. ${ }^{5}$ One of the earliest formulations of this problem was presented by Leissa, who tabulated a frequency parameter for four vibration modes of simply supported circular plates with varying rotational stiffness. ${ }^{1}$ Kang and Kim presented an extensive review of the modal properties of elastically restrained beams and plates. ${ }^{6}$ Zheng and Zhou studied the large deflection of a circular plate resting on a Winkler foundation. ${ }^{7}$ Ghosh studied the free and forced vibration of circular plates on a Winkler foundation by an exact analytical method. ${ }^{8}$

The most general soil model used in practical applications is the Winkler model in which the elastic medium below a structure is represented by independent linear elastic springs. ${ }^{9}$ Recent investigations have reiterated the efficiency of the classical approach in analyzing the behavior of structures under vibrations. ${ }^{10}$ Although the vibration of circular plates with elastic edge support and resting on an elastic foundation is of considerable interest, the availability of extensive data is severely limited; it is difficult to establish potential data trends with limited data available. Therefore, in this study, 12 vibration modes are studied. The present study considers the problem of vibrations of circular plates with an elastically restrained edge against rotation and resting on a Winkler foundation. 